\title{
A Case Report of Cervical Embroynal Rhabdomyosarcoma Treated with Multimodality Approach
}

\author{
Dr. Mridul Anand ${ }^{1,}$ Dr. Ankita Parikh ${ }^{2,}$ Dr. Mitul Modi ${ }^{3}$ \\ Department ofRadiation Oncology, Gujarat Cancer Research InstituteAhmedabad, India
}

\begin{abstract}
Embryonal rhabdomyosarcoma of the female genital tract is a rare and highly malignant entity. It is usually diagnosed in adolescence, in contrast to vaginal rhabdomyosarcoma, which occurs mostly in infants. With the advent of multimodality treatment, prognosis seems to be improved. We report a case of a 20 -year old young female presenting with a protruding mass from vaginal introitus and irregular bleeding. Polypectomy was done and microscopic findings were confirmed as a cervical embryonalrms.Neoadjuvant chemotherapy showed excellent response, after which Total Abdominal Hysterectomy and bilateral Lymphadenectomy was done. Histopathology showed embroyonalrhabdomyosarcoma involving cervical lip, infiltrating more than half thickness of cervical wall.Patient was treated with post-operative radiotherapy and she is on regular follow-up. The presence of a cervical polyp in an adolescent is a gynecological oddity and should be histologically examined. The prognosis of the cervical embryonal RMS depends on clinical and histological features. The current treatment protocols are based on trials.Patients with unfavorableprognosis characteristics, the multimodality approach including surgery, adjuvant chemo and radiotherapy can be effective. Otherwise, surgery alone can be effectively proposed.
\end{abstract}

Keywords: Botryoidrms, Cervicalrms, Embryonal soft tissue sarcoma, cancer cervix presenting as polyp, Young female with recurrent polyp.

\section{Introduction}

Rhabdomyosarcoma (RMS) is a tumor of skeletal muscle, which is classified by WHO into Embryonal, Alveolar, Pleomorphic and spindle cell/sclerosing RMS subtypes. It is the commonest soft tissue sarcoma in childhood and young adults, accounting for 4 to $6 \%$ of all malignancies in this age group. It is a highly malignant tumor arising from embryonal mesenchyme.Due to rarity of cervical RMS, there is limited literature of only a few case reports, in which appropriate line of management is not outlined. We report a case of embryonal RMS that presented as a cervical polyp in a young female, treated with neoadjuvantchemotherapy followed by hysterectomy and post-operative radiotherapy and adjuvant chemotherapy.

\section{Case}

An unmarried 20 year old female patient, presented to gynecological opd of our hospital in may 2014 with chief complaints of irregular bleeding since 5 months and feeling of something coming out of vagina since 3 months.

\subsection{Past History}

Patient had similar complaints 1 year back for which she underwent cervical polypectomy outside. Histopathology was suggestive of simple cervical polyp of size $2.5 * 2.8 \mathrm{~cm}$. After few months, symptoms recurred and repeat polypectomy was done in nov.2013. Histopathology was suggestive of sessile polyp $3.5 * 2.5$ $\mathrm{cm}$ arising from posterior cervical lip.

\section{2present History}

The patient had similar complaints again after 6 months.On gynecological examination, irregular and fleshy elongated polyp was seen at the vulvar orifice with necrotic tip. The mass had multiple nabothiancyst like areas on it. It was not friable and did not bleed on touch. On $\mathrm{p} / \mathrm{v}$ examination, the mass was sessile arising from the elongated anterior lip of the cervix. On per rectal examination, cervix was normal transversely and parametriumwas free.

\subsection{Histological Diagnosis}

Slides and blocks were sent for review at two different centers and Imaging tests were ordered. In the slide review of the outside laboratory, Myogenin\& Desmin (RMS marker) were positive and CD 10 (endometrial stromal sarcoma marker) was found to be negative. Possibility of adenosarcoma with rhabdomyoblastic differentiation or botryoid rms was favoured. Opinion at higher center suggested embroynal rms.Biopsy reviewed at our institute was suggestive of of embryonal rhabdomyosarcoma,botryoid subtype. 


\subsection{Imaging}

MRI (May 2014)- 45*22 mm lesion in axial plane with craniocaudal extension of $73 \mathrm{~mm}$, well defined lobulated soft tissue lesion arising from cervix and showing exophytic component extending caudally and projecting into vagina with no evidence to extension to pelvic wall.

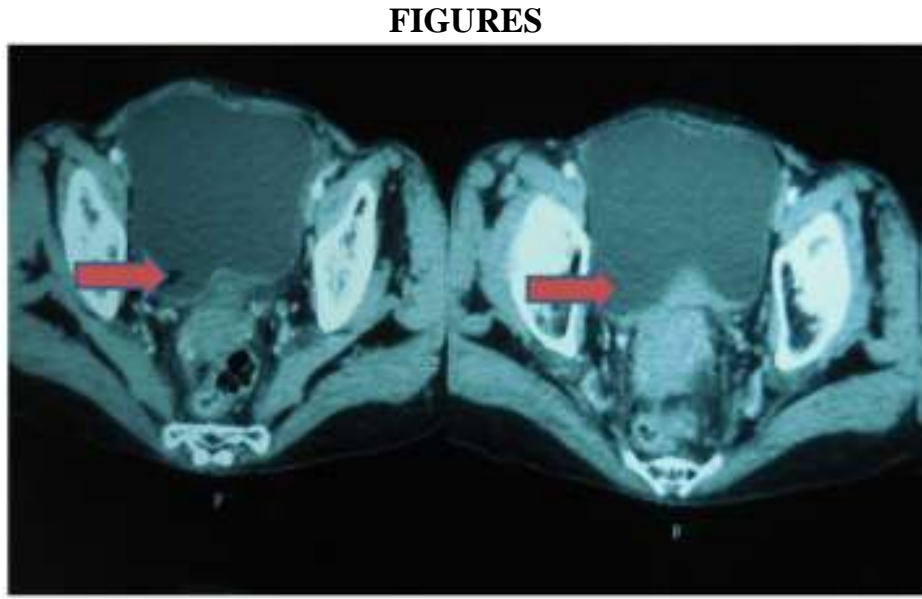

Figure 1- Cervical mass extending to vagina

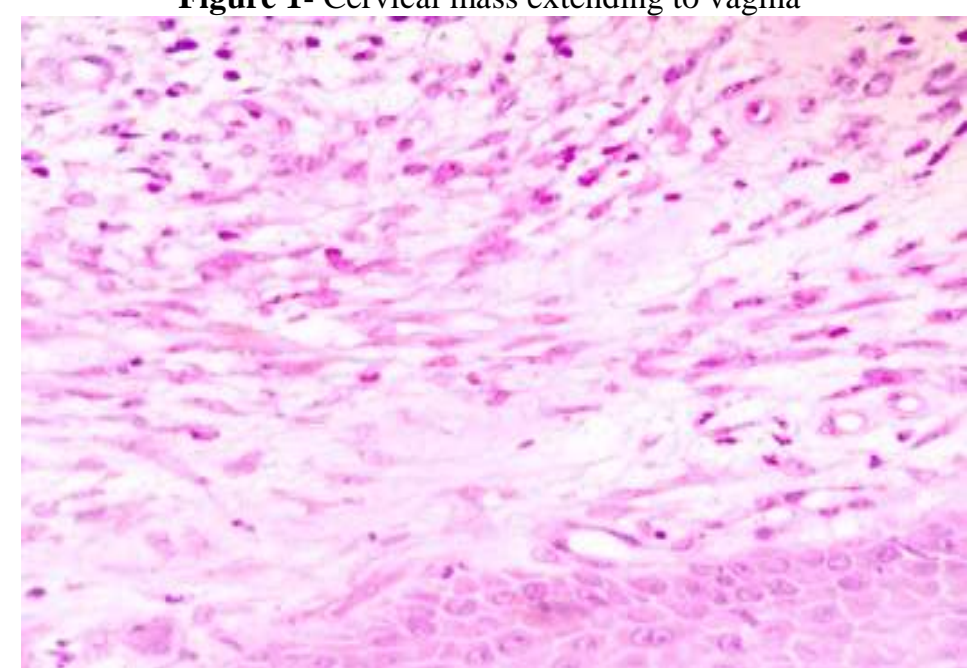

Figure 2- Histological picture- characterstic round cells

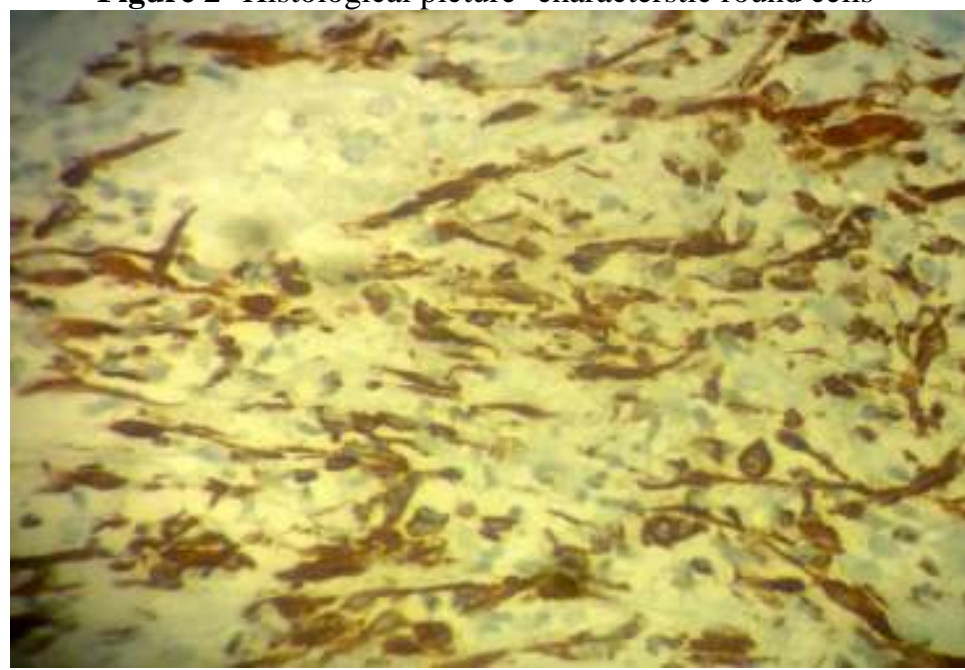

Figure 3- Desmin Positive 

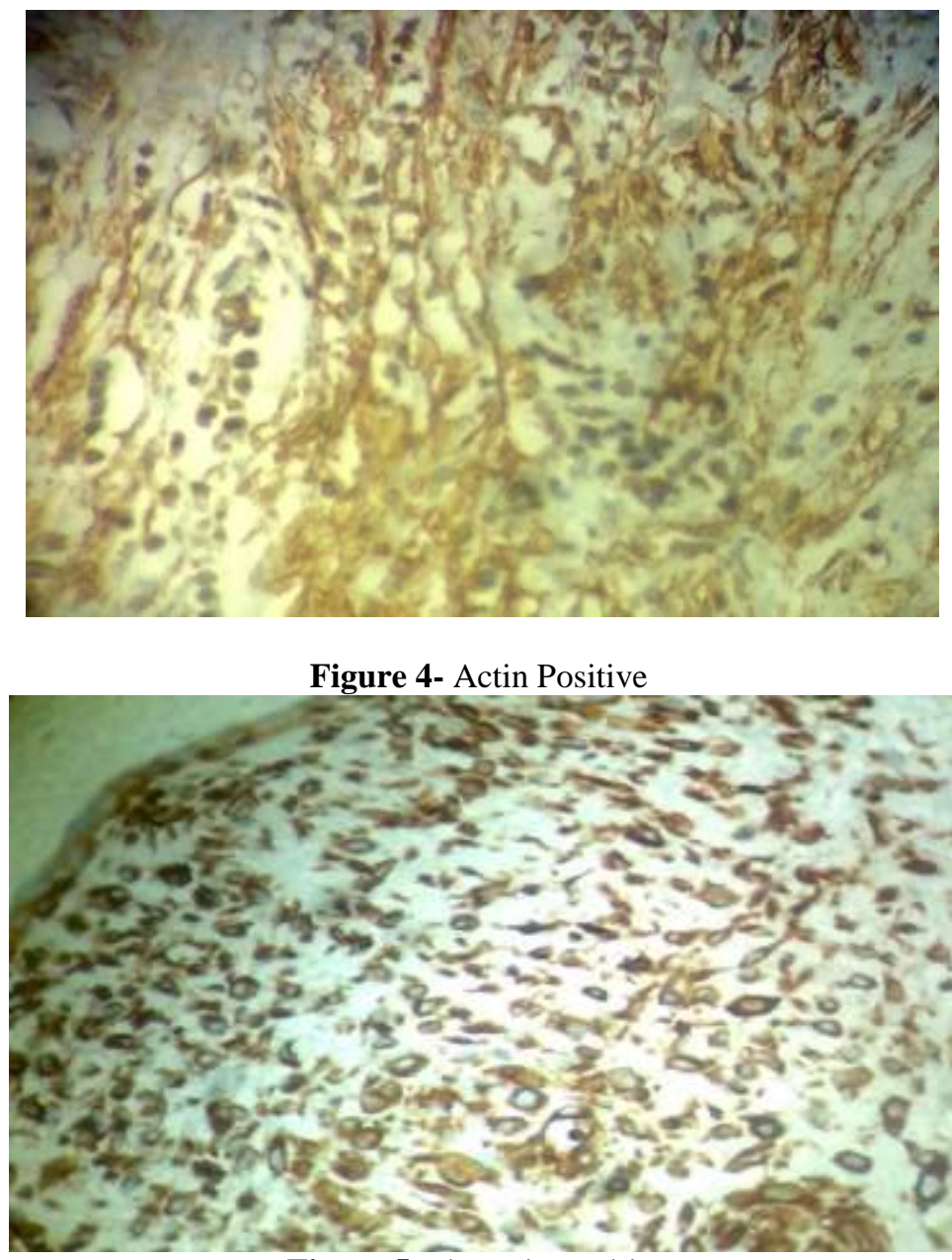

Figure 5-Vimentin Positive

\subsection{Treatment}

Patient was planned for neoadjuvant chemotherapy on VAC protocol. The first cycle was given on $2 / 6 / 14.50 \%$ clinical response was seen after first cycle and $2^{\text {nd }}$ cycle on $25 / 6 / 14$. Likewise $5^{\text {th }}$ cycle every 3 weekly was completed on $12^{\text {th }}$ September 2014.Post chemotherapy showedbulky cervix with mild eccentric wall thickening on right side, possibilityof mild residual lesion could not be excluded. No evidence of liver metastasis or paraaoric lymph nodes was noted. Before planning for surgery, post-chemotherapy examination under anaesthesia was done. Examination showed excellent response to chemotherapy; with disease involving only base,probably arising from ectocervix at about $10^{\prime} 0$ clock position. Posterior lip was completely flushed with vagina.

One more cycle of chemotherapy was planned (week 12) was received on $12^{\text {th }}$ sept. $\left(5^{\text {th }}\right.$ cycle).Exploratory laparotomy followed by Total Abdominal Hysterectomy and bilateral Pelvic Lymphadenectomy with Left ovarian cyst biopsy and lower para-aortic lymph node sampling and bilateral ovarian transposition was done. Histopathology showed residual tumor -Amboyna Rhabdomyosarcoma (spindle cell type) involving mainly anterior lip of cervix, infiltrating more than half thickness of cervical wall. Tumor measuring-2.9*2.5*1.6 Cc and vaginalfornices, lower part of uterus, endometrium \& myometrium and resection margins were free of tumour.13 Pelvic nodes were dissected and none was found to be positive. Single paraaortic was dissected and was negative. Post-operative $6^{\text {th }}$ cycle adjuvant chemotherapy (VCR) was given. Before proceeding with radiotherapy, thorough gynecological examination was done and found to be negative.

Patient was planned for post-operative Radiotherapy by 3D-CRT ( $50 \mathrm{GY} / 25 \#)$ followed by 2 sessions of intracavitary brachytherapy (CVC) 6.5 Gy each 1 week apart. Post-operative radiotherapy was completed without any complications. This was followed by completion of adjuvant chemotherapy in the form of Vincristine, Actinomycin and Cyclophosphamide till week 43.The entire treatment was completed in Oct. 2015. The patient has been kept on observation with imaging studies at regular intervals and is disease free at present.

\section{Discussion}


Rhabdomyosarcoma has been classified into three major histological types by the International RMS Study Group (IRS) :Embryonal, alveolar and undifferentiated. There is a variant of Embroyonal type, Botryoid type, which has a typical 'grape-like' appearance. The most common sites of origin for the embroyonal RMS are the head and neck, genitourinary and the extremities. In the genitourinary site, vagina is the most common site, occurring in the first decade of life. Only $0.5 \%$ of primary RMS in girls is found in cervix. In contrast to the vaginal RMS, age at presentation is $2^{\text {nd }}$ decade occurring in young females.In a small proportion of the patients, genetic association with Li-Fraumeni syndrome has been found.

Most patients present with a feeling of a mass in introitus.It may present as a soft,grape-like clusters, as single or multiple polyps, as in our patient.Other symptoms include leucorrhea, bleeding and vaginal discharge. Gross examination showed tumors having polypoid appearance with grape-like smooth and glistening structures. On microscopic examination, rhabdomyoblastic nature of the tumor cells was noted by the smallrounded tumor cells. Differential diagnosis includes benign conditions like rhabdomayoma, pseudosarcomabotryoides and malignant conditions like adenosarcoma.Primary RMS of cervix, due to its rarity, lacks any superior treatment protocols from any single institution. However, treatment of genitourinary primary RMS can be extrapolated. The use of multidrug chemotherapy regimen as adjuvant to surgery has improved the overall prognosis in several trials. Management of RMS of the female genital tract has now been evolved towards preservation of genitourinary tract, due to recent reports that sarcoma botryoid of cervix behaves less aggressively than that of vagina. Studies $(1,2)$ comparing aggressive surgery and radiation with chemotherapy have reported similar survival, while maintaining fertility. IRSG $(3,4)$ states that fertility sparing surgery followed by chemotherapy is the appropriate treatment for local control. However, there are case reports of unfavorable outcomes with this management.

The prognostic factors include histological type(embryonalbotryoid having favourable outcome than alveolar subtype), metastatic disease at presentation and poor response to chemotherapy. The extent of the disease following primary surgical procedure is the most important prognostic factor.

In the IRSG3, patients with clinical group I (no residual tumor after surgery), Clinical group II (microscopic residual post-surgery) and Clinical group III (gross residual surgery) had a 5-year survival of $90 \%, 80 \%$ and $70 \%$ respectively.

\section{Conclusions}

Cervical RMS is a rare tumor occurring in young females and should be included in the differential diagnosis of a large cervical polyp, presenting with vaginal bleeding. An early diagnosis is important as it favors a good prognosis. Surgery and chemotherapy is the mainstay of treatment with multimodality treatment having good outcomes.

\section{References}

[1]. Riesman ML, Wang W-L et al, Rhabdomyosarcoma of the cervix in adult women and younger patients. Gynecoloncol. 2012, 126:351-356

[2]. Viella et al: Rhabdomyosarcoma of the cervix in sisters with review of the literature. GynaecolOncol 2005, 99: 742-748

[3]. Zrara I etall :Botryoid sarcoma of the uterine cervix. GynecologieObstetriqueFertilite 2002, 30:784-786

[4]. Kayton ML, Wexler LH et all: Pediatric radical abdominal trachelectomy for anaplastic embryonalrhabdomyosarcoma of the uterine cervix; an alternative to radical hysterectomy. J PediatrSurg 2009, 44:862-867. 